\title{
DETERMINACIÓN DE PROPIEDADES FÍSICO-QUIIMICAS DE LOS MATERIALES AGREGADOS EN MUESTRA DE ESCOMBROS EN LA CIUDAD DE BOGOTÁ D. C.
}

\author{
Álvaro Chávez Porras \\ Nataly Lorena Guarín Cortes* \\ María Carolina Cortes Duarte ${ }^{* * *}$
}

Recibido: 04/06/2012

Aceptado: 07/05/2013

\section{RESUMEN}

Con la implementación de operaciones unitarias apoyadas en ensayos de laboratorios y evaluando propiedades físico-químicas, se concertó en la necesidad de adoptar estrategias dirigidas a la reducción, reúso y reciclaje de los escombros en la ciudad de Bogotá D. C. Lo anterior permite aumentar la vida útil de los rellenos sanitarios del área, reducir la explotación de los recursos naturales y la indiscriminada disposición en lugares no autorizados. Con una muestra local, este estudio identificó las características como: granulometría; absorción; porosidad; resistencia a la compresión; $\mathrm{pH}$; carbono orgánico total; metales pesados y elementos menores. Resultados de experiencias, nacionales e internacionales, se compararon con esta y se concluyó que los componentes de estos agregados tienen semejanza a los obtenidos de forma natural; ya que poseen alto potencial para ser utilizados en componentes de construcción civil sin función estructural, y cumplen con las especificaciones de las Normas Técnicas Colombianas - NTC.

Palabras clave: agregados, reciclaje, propiedades físico-químicas de escombros.

Ing. industrial, doctor en Ingeniería Civil, Área Ambiental. Profesor asistente, Facultad de Ingeniería. Líder Grupo Producción, Innovación y Tecnología, Universidad Militar Nueva Granada, Bogotá D. C. Colombia. Carrera 11 N.`101-80. Tel: 6500000 Ext. 1716. alvaro.chavez@ unimilitar.edu.co

** Ingeniera industrial. Joven y asistente de Investigación Grupo Producción, Innovación y Tecnología, Facultad de Ingeniería, Universidad Militar Nueva Granada, Bogotá D. C. Colombia. Carrera 11 N.101-80. Tel: 6500000 Ext. 1716. nataly.guarin@unimilitar.edu.co

*** Ingeniera química, Facultad de Ingeniería, Auxiliar de Investigación, Facultad de Ingeniería. Grupo Producción, Innovación y Tecnología, Universidad Militar Nueva Granada, Bogotá D. C., Colombia. ingenieraquimik22@hotmail.com 


\title{
DETERMINATION OF PHYSICAL-CHEMICAL PROPERTIES OF THE MATERIALS ADDED IN SAMPLE DEBRIS IN THE CITY OF BOGOTÁ D.C.
}

\begin{abstract}
With the implementation of unit operations supported by laboratory tests, by evaluating physical and chemical properties, was concluded on the need to adopt strategies to reduce, reuse and recycling of debris in the city of Bogotá D.C. This can increase the landfills lifespan, reduce the exploitation of natural resources and the indiscriminate disposal in unauthorized places. With a local sample, some characteristics as granulometry; absorption; porosity; compressive strength; $\mathrm{pH}$; total organic carbon; heavy metals and trace elements were identified. Results of experience, national and international, were compared, concluding that the components of these aggregates have similarity to those obtained naturally, because they have high potential for use in civil construction components without structural function, in compliance with the Colombian Technical Standards - NTC.
\end{abstract}

Key words: Aggregates, recycling, chemical and physical properties of debris. 


\section{INTRODUCCIÓN}

El aumento demográfico mundial y la insuficiencia de infraestructuras que respondan al desarrollo de las economías de los centros urbanos, tanto como la falta, en muchos casos, de políticas que tiendan al saneamiento básico, sumado al deterioro ambiental, actualmente conforman la problemática de la cantidad de residuos sólidos de construcción y demolición civil - RCD.

Hoy en día, las sociedades en desarrollo poseen un efecto dinamizador en lo que respecta a la demanda de insumos para la construcción civil, como el cemento, ya que se manejan tasas de consumo del orden de $300 \mathrm{~kg} / \mathrm{hab} / \mathrm{año}$, razón que hace evidente la adopción de estrategias dirigidas a la reducción, reciclaje y reutilización de los escombros -RCD- [1]. Se resalta en el ámbito nacional la producción actual per cápita de cemento, que supera lo reportado en el 2005 por el Instituto Colombiano de Producción de Cemento -ICPC-, con $170 \mathrm{~kg} /$ hab./año [2].

Las disposiciones legales nacionales, imputadas a los procesos de planeación y gestión ambiental, se dirigen en el desarrollo sostenible a la planificación, ejecución y control de las politicas ambientales en los procesos de producción. Además, en Colombia, la Ley 152 de 1994, Ley Orgánica del Plan de Desarrollo, establece herramientas para la formulación de políticas ambientales locales, municipales, departamentales y de orden nacional en los diferentes planes [3]. Existe también, la Ley 142 de 1994, que genera responsabilidades a los municipios para certificar la óptima prestación de los servicios públicos domiciliarios [4]. En esa búsqueda de soluciones, el país está en la exploración de metodologías sostenibles, que permitan mitigar el impacto negativo que los RCD causan sobre el medioambiente.

Con todo lo anterior, en el proyecto se evaluaron las propiedades físico-químicas, para identificar la viabilidad de uso y generar nuevas alternativas en cuanto a la preclasificación, correcta disposición y reincorporación en los sistemas productivos; donde se presenta la incorporación de operaciones unitarias de agregados reciclables, sobre la base de información recopilada, de experiencias nacionales e internacionales.

En la caracterización de la muestra de RCD, bajo sus respectivos ensayos de laboratorio, se identificaron estas principales propiedades, como lo son: granulometría, porcentaje de absorción, porosidad, resistencia a la compresión, $\mathrm{pH}$, carbono orgánico total, metales pesados (aluminio$\mathrm{Al}$, plomo-Pb, cadmio-Cd, níquel-Ni y azufre-S), elementos menores (hierro-Fe, manganeso-Mn), sulfatos y silicatos.

Basados en la comparación de análisis, con los provistos en la literatura, se establece que los RCD son una fuente potencial de materia prima para la fabricación de componentes de construcción civil sin función estructural, al obtener principalmente resultados promedio de $8,1 \%$ de absorción y 39,3 $\mathrm{kg} / \mathrm{cm}^{2}$ de resistencia a la compresión para un tiempo de fraguado de 28 días.

\section{MARCO REFERENCIAL}

Los RCD constituyen una mezcla heterogénea de varios elementos como ladrillo, cemento, agregados (arena, grava, gravilla), recebo, acero, madera y otros, que dificultan su recuperación o reincorporación a determinado ciclo económico y/o productivo [5].

Están constituidos por $20 \%$ de concreto, 50 $\%$ de material de albañilería (cerámico, mortero, ladrillos, etc.), $10 \%$ de asfalto y $20 \%$ de otros elementos como maderas y partes metálicas [6]. Sin embargo, cifras de demolición afirman que el concreto conforma las $3 / 4$ partes de los residuos de esta actividad, lo que indica la importancia de su estudio y la búsqueda de nuevas metodologías para su aprovechamiento [7].

Estudios establecieron que alrededor del 64 $\%$ de los residuos procedentes del municipio de Campinas-Brasil, dirigidos a la Unidad Recicladora de Materiales São Caetano, se componen de 


\section{CONSOLIDADO DE BOGOTÁ D.C. PESO POR MATERIAL (t)}

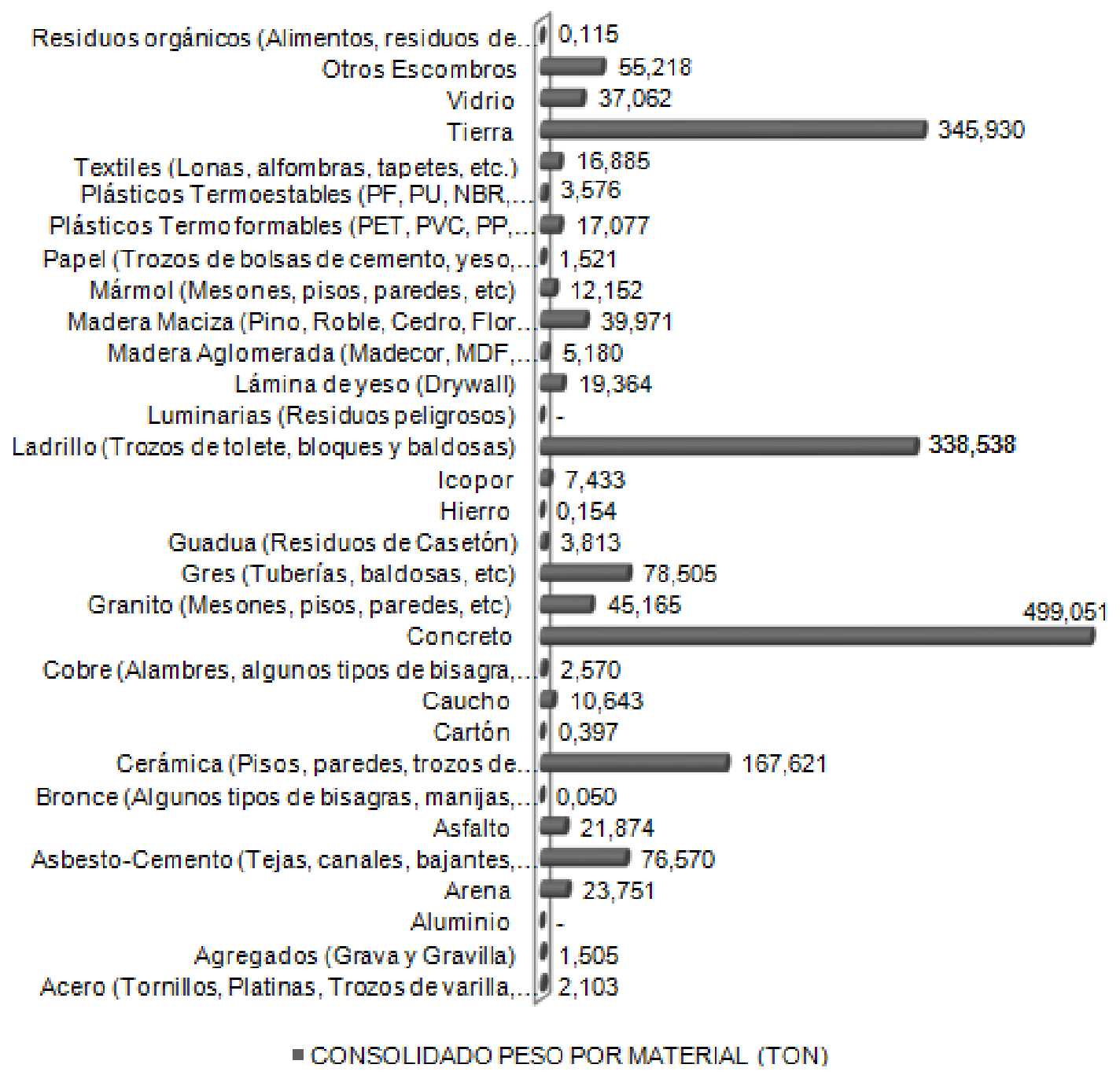

Figura 1. Consolidado de la caracterización por toneladas de escombros de Bogotá D. C. Fuente: [8]

concreto, $30 \%$ de elementos de mampostería y el $6 \%$ restante de otros materiales (mortero, piedra, arena, metales y plásticos) [5]. Esta composición, presentada en la tabla 1 , guarda semejanza con el panorama nacional colombiano, de acuerdo con un estudio practicado por la Unidad Administrativa Especial de Servicios Públicos del Distrito Capital - UAESP [8]. En este, se evidenció que la ciudad de Bogotá D. C. cuenta con puntos críticos y temporales, donde se generan y/o destinan toneladas de escombros. Se realizó una recolección de 133 muestras, con un peso total de 1813 t representadas en $1912 \mathrm{~m}^{3}$; donde se identificaron las siguientes cantidades representativas: concreto, 499 t; ladrillo, 399 t; tierra 346 t, y cerámica, 79 t. Los resultados de esta caracterización se observan en la Figura 1, la cual se comparó con los resultados obtenidos en el manejo del municipio de Campinas- Brasil, [5], presentados en la Figura 2. 


\section{COMPOSICIÓN DE ESCOMBROS MUNICIPIO DE CAMPINAS-BRASIL}

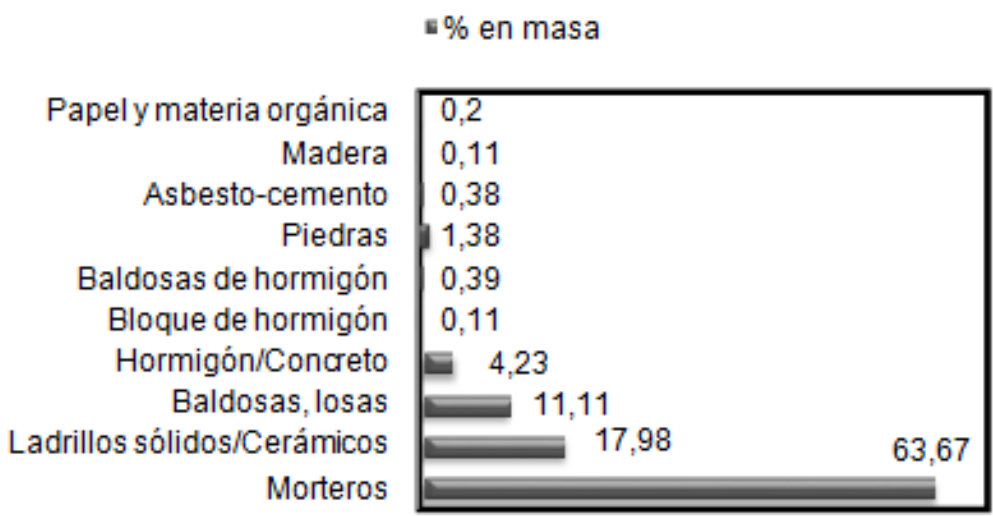

Figura 2. Consolidado en masa de los escombros Campinas, Brasil.

Fuente: Chávez [5]

Tabla 1. Composición media de residuos de construcción

\begin{tabular}{|l|c|}
\hline \multicolumn{1}{|c|}{ Elemento } & \% en masa \\
\hline Concretos & 63,67 \\
\hline Ladrillos sólidos & 17,98 \\
\hline Baldosas, losas & 11,11 \\
\hline Mortero & 4,23 \\
\hline Bloque de hormigón & 0,11 \\
\hline Baldosas de hormigón & 0,39 \\
\hline Piedras & 1,38 \\
\hline Asbesto-Cemento & 0,38 \\
\hline Madera & 0,11 \\
\hline Papel y materia orgánica & 0,20 \\
\hline
\end{tabular}

Fuente: [5]
En general, los agregados son minerales sólidos inertes, que con granulometrías adecuadas se convierten en materia prima de otros productos artificiales, y su mezcla con aglomerantes de activación hidráulica como el cemento o la cal, le otorga una resistencia adicional [9]. Se afirma que estos constituyen más del $70 \%$ de cada $\mathrm{m}^{3}$ de concreto fabricado, lo que confirma lo imprescindibles que pueden llegar a ser estos materiales [10]. No poseen una clasificación particular, ya que se pueden categorizar de acuerdo con su origen, composición, forma de partícula, color, modo de fragmentación o tamaño de partícula. Sin embargo, la procedencia es la forma más común de identificarlos, por la cual se dividen en tres grupos: naturales, superficiales

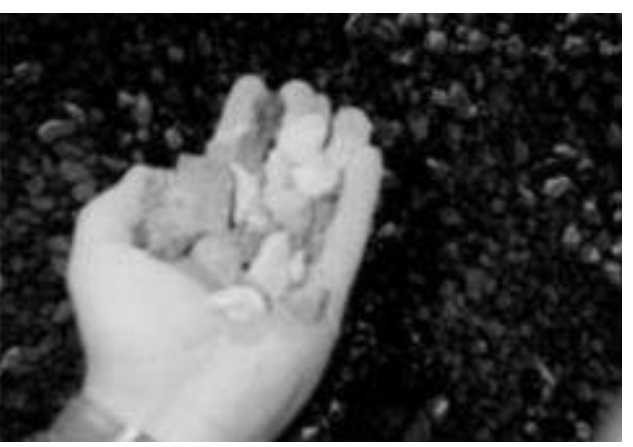

Figura 3. Agregados reciclado utilizado para la elaboración de concreto

Fuente: [11] 
Tabla 2. Aplicaciones de los agregados reciclados según su procedencia

\begin{tabular}{|l|c|l|}
\hline \multicolumn{1}{|c|}{ Materiales } & Contenido & \multicolumn{1}{c|}{ Aplicaciones } \\
\hline Cerámicos & $>90 \%$ & $\begin{array}{l}\text { Materiales de relleno, pistas forestales, jardinería, cubiertas ecológicas y aplicaciones } \\
\text { deportivas (tenis, tierra batida) }\end{array}$ \\
\hline Concreto & $>90 \%$ & $\begin{array}{l}\text { Materiales de relleno y recubrimiento, bases y sub-bases de carretera, agregados para } \\
\text { concretos y agregados para materiales con ligantes. }\end{array}$ \\
\hline Materiales pétreos & $>90 \%$ & Materiales de relleno, zahorras y materiales para muros y aplicaciones acústicas. \\
\hline
\end{tabular}

Fuente: [12]

y reciclados [11]. La Figura 3 ilustra algunos agregados reciclados, utilizados como materia prima potencial para la elaboración de concreto.

Las aplicaciones de los agregados reciclados dependen principalmente de la composición del residuo original. La tabla 2 resume estas aplicaciones teniendo en cuenta la cantidad de material cerámico, concreto o material pétreo presente en el residuo de procedencia [12]. Experiencias desarrolladas en el mundo contribuyen a la identificación de la matriz o composición fisicoquímica que predomina en estos materiales.

Respecto a acciones de gestión, en el ámbito internacional, decisiones estratégicas como las de Nueva Zelanda, Estados Unidos, Irlanda, México, Brasil y España, evidencian la necesidad de instalar plantas de tratamiento y recuperación que otorguen valor agregado a los materiales que puedan obtenerse como producto de los procesos de reciclaje [13].

El Gobierno irlandés creó en el año 2001, el Consejo Nacional de Desechos de la Construcción y Demolición, identificado por sus siglas en inglés NCDWC, el cual proporciona un marco para lograr el cumplimiento de las políticas y los objetivos establecidos por el Ministerio de Medio Ambiente y el Gobierno local; en consecuencia, elaboran planes de acción y asesoría en cualquier tema relacionado con los RCD, incluyendo recomendaciones y medidas que podrían adoptarse para asegurar el cumplimiento de los requisitos legales [14].

Desde el año 2002, el Gobierno de Nueva Zelanda ha establecido diferentes mecanismos para reducir en un $50 \%$ la disposición de escombros en rellenos sanitarios, realizando promoción de las guías REBRI (Resource Efficiency in Building and Related Industries) dirigidas a las firmas o compañias responsables de las actividades de construcción y demolición [13].

España, por su parte, cuenta con avances notorios en cuanto a la gestión de los RCD, evidenciados mediante la instalación y puesta en marcha de varias plantas de clasificación y tratamiento de escombros [15].

En Francia, existe la Unión Internacional de Laboratorios y Expertos en Materiales de Construcción, Sistemas y Estructuras -RILEM-, que en 1994, en el TC121-DRG, recogió una serie propiedades físicas y químicas que deben tenerse en cuenta para la reutilización de agregados reciclados, clasificándolos según la tabla 3 en tres grandes tipos: tipo I (procedentes de escombros de albañilería y/o mampostería), tipo II (procedentes de escombros de concreto) y tipo III (mezcla de agregados reciclados y agregados naturales o tradicionales )[16].

La Unión Europea cuenta con legislación específica para este tipo de materiales; por ejemplo, la directiva 1993/31/CE establece las condiciones del vertido, mientras que la directiva 2000/532/ CE consiste en el Catálogo Europeo de Residuos (CER). En cada una de estas se denotan los RCD de manera genérica, en cuatro categorías que corresponden a concreto, albañilería, asfalto y otros $[17,18]$.

El panorama latinoamericano refleja la ausencia de avances tecnológicos y políticos en compa- 
ración con la Comunidad Europea, no solo por su inminente desarrollo sino en gran parte, por la regulación que tienen los gobiernos para ejercer control y seguimiento a los RCD, desde su generación hasta su disposición final [19].

Brasil constituye el primer país latinoamericano que garantiza la adecuada gestión de escombros, gracias a la Resolución 307 expedida por CONAMA (Consejo Nacional del Medio Ambiente) en el 2002, la cual establece modelos de acción para el reciclaje; allí, distintas ciudades como São Pablo y Campinas cuentan con unidades de aprovechamiento y tratamiento de escombros [20].

México, desde el 2002, encabeza el segundo lugar con la operación de «Concretos Reciclados S. A.», la primera planta de RCD del Distrito Federal, que recibe 4000 t/día. El tratamiento de los agregados guarda similitud con los procesos llevados a cabo por la Comunidad Europea [19, 20].

En Colombia, el desarrollo de tecnologías dispuestas para la clasificación de escombros y/o metodologías para la fabricación de agregados reciclados es casi nula; sin embargo, algunas insti- tuciones educativas, públicas y privadas, han tenido iniciativas para diseñar lineamientos a favor de ciudades libres de escombros, como son los casos de Bogotá y Medellín [8].

Respecto a la composición de los RCD [5], la ciudad de Campinas-Brasil sugirió separar los suelos, las arenas y los materiales gruesos de los escombros; los dos primeros destinados a la cobertura en vertederos, mientras los últimos destinados a la trituración y cribado de acuerdo con su composición o matriz dominante, llamadas: matriz cerámica AG-CER-URM (agregado reciclado matriz cerámica URM); matriz concreto AGCON-URM (agregado reciclado matriz concreto URM) y mezcla AG-MIS-URM (agregado reciclado matriz de mezcla), donde se aclara que las propiedades físicas y químicas dependen directamente de la procedencia y aplicabilidad, y se manifiesta que las especificaciones técnicas acerca de su uso difieren para morteros, cerámicos, ladrillos y bloques [5].

En la lista de las propiedades identificadas por RILEM TC121 [16], que se deben tener en cuenta

Tabla 3. Clasificación y especificaciones de los agregados reciclados en concretos según RILEM

\begin{tabular}{|l|c|c|c|c|}
\hline \multicolumn{1}{|c|}{ Características } & \multicolumn{3}{|c|}{ Agregados gruesos reciclados para el concreto } \\
\cline { 2 - 5 } & Tipo I & Tipo II & Tipo III & Métodos de ensayo \\
\hline Densidad mínima de partículas secas $\left(\mathrm{kg} / \mathrm{m}^{3}\right)$ & 1.500 & 2.000 & 2.400 & ISO $6783 \mathrm{y} 7033$ \\
\hline Máxima absorción de agua $(\% \mathrm{p} / \mathrm{p})$ & 20 & 10 & 3 & ISO $6783 \mathrm{y} 7033$ \\
\hline Contenido máximo de material con: SSS<2.200 $\mathrm{kg} / \mathrm{m}^{3}(\% \mathrm{p} / \mathrm{p})$ & - & 10 & 10 & ASTM C123 \\
\hline Contenido máximo de material con: SSS $1.800 \mathrm{~kg} / \mathrm{m}^{3}(\% \mathrm{p} / \mathrm{p})$ & 10 & 1 & 1 & ASTM C123 \\
\hline Contenido máximo de material con: SSS<1.000 $\mathrm{kg} / \mathrm{m}^{3}(\% \mathrm{p} / \mathrm{p})$ & 1 & 0,5 & 0,5 & ASTM C123 \\
\hline Contenido máximo de impurezas: $(\mathrm{bitumen}, \mathrm{vidrio}, \mathrm{plástico})(\%)$ & 5 & 1 & 1 & Visual \\
\hline Contenido máximo de metales $(\%)$ & 1 & 1 & 1 & Visual \\
\hline Contenido máximo de materia orgánica $(\%)$ & 1 & 0,5 & 0,5 & NEN 5933 \\
\hline Contenido máximo de finos $(<0,063 \mathrm{~mm})(\%)$ & 3 & 2 & 2 & PrEN $933-1$ \\
\hline Contenido máximo de arena $(<4 \mathrm{~mm})(\%$ en peso) & 5 & 5 & 5 & PrEN 933-1 \\
\hline Contenido máximo de sulfatos $(\% \mathrm{p} / \mathrm{p})$ & 1 & 1 & 1 & BS 812 part- 118 \\
\hline
\end{tabular}

Fuente: [9] 
para el uso de agregados reciclados en estructuras a base de concreto se enuncian: granulometría, forma y textura superficial, absorción, mortero adherido, densidad, resistencia a la abrasión. La lista de propiedades con sus respectivas especificaciones se describe en la tabla 3.

Por otra parte, el conocimiento de las propiedades químicas de los agregados permite considerar la concentración de contaminantes con alto nivel de toxicidad, que puedan causar deterioro en el medioambiente y perjudicar los procesos de reciclaje. Algunos materiales como polímeros, yeso, asbesto, cloruros, materia orgánica, metales pesados, vidrio, aluminio, entre otros, son evaluados mediante ensayos de laboratorio como: $\mathrm{pH}$, sulfatos, humedad, densidad, carbono orgánico -CO-, sólidos volátiles y metales pesados. [5].

\section{METODOLOGÍA}

Se propuso una metodología de actividades y procedimientos, basada en el análisis de las propiedades físico-químicas de las matrices cerámica y concreto, en función del potencial que tienen los RCD para ser recuperados.

La Figura 4 incluye la implementación de operaciones unitarias apoyadas en la aplicación de ensayos de laboratorio para cada uno de los materiales a reciclar; siguiendo las especificaciones del Instituto Colombiano de Normas Técnicas y Certificación -ICONTEC-y el Instituto Nacional de Vías de Colombia -INVIAS- [21, 22].

La fabricación de nuevos productos en función del potencial recuperable depende de los agregados y sus características físico-químicas, y la incorporación de operaciones unitarias depende, además, de la capacidad de la planta. La Figura 5 resume las unidades básicas que requiere una planta fija, recomendada para producir materiales reciclados de fácil aplicación en obras de construcción, reduciendo el volumen de los RCD destinados al relleno sanitario, a partir de su cribado y trituración permanente [16].

Los resultados físico-químicos analizados incluyeron ensayos como: \% Si, \% Al, $\mathrm{Cr}(\mathrm{mg} / \mathrm{kg})$, $\mathrm{Pb}(\mathrm{mg} / \mathrm{kg}), \mathrm{Cd}(\mathrm{mg} / \mathrm{kg}), \% \mathrm{C}$ (Total), \% N (To-

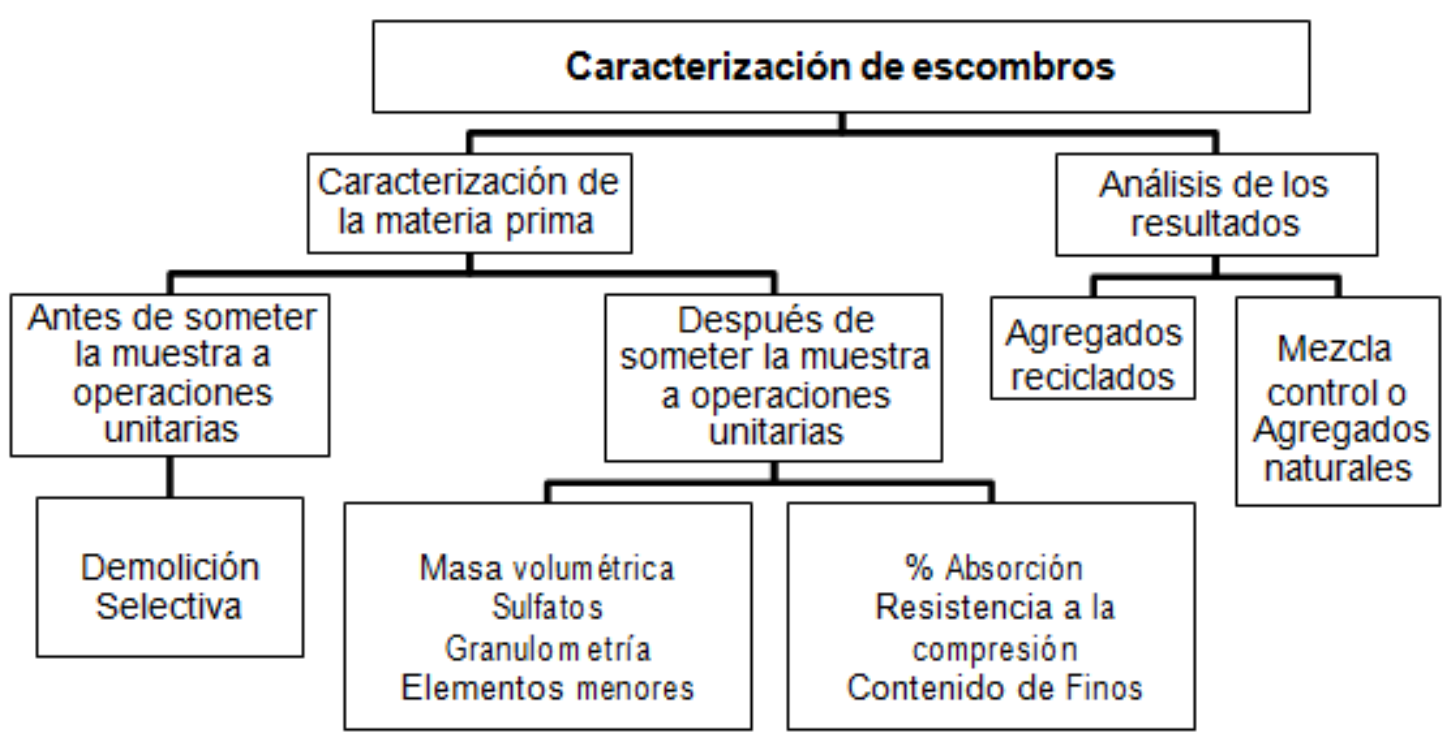

Figura 4. Resumen de la metodología propuesta para el reciclado de escombros

Fuente: $[21,22]$ 


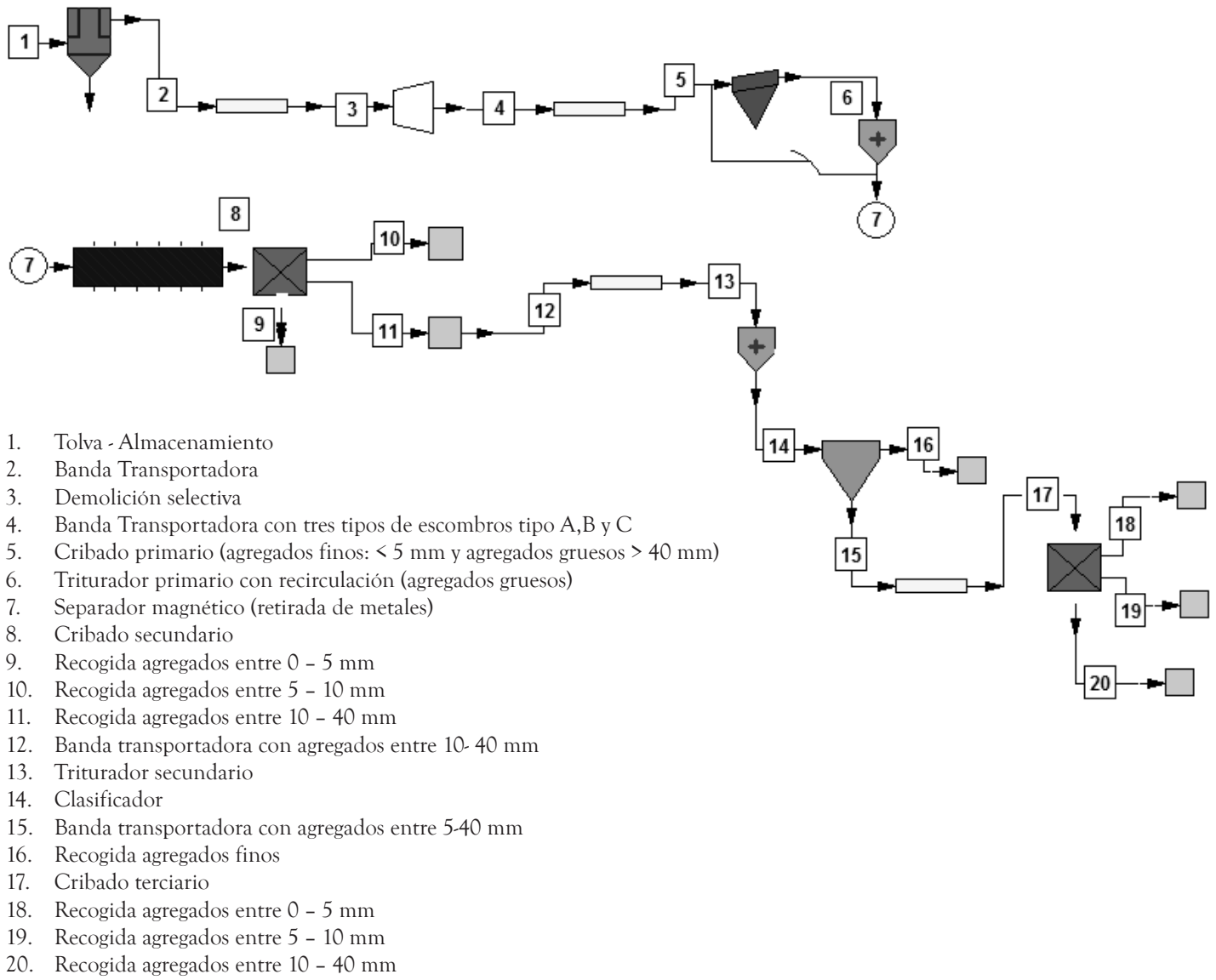

Figura 5. Resumen montaje planta de RCD

Fuente: [16]

tal), sulfatos, materia orgánica $-\mathrm{MO}-$, elementos menores y granulometría. La tabla 4 muestra la compilación de los métodos de evaluación.

Una vez identificada esta metodología, el desarrollo del proyecto inició con la recolección de escombros en distintos puntos de generación en la ciudad de Bogotá D. C. Consecuentemente, se procedió a la trituración y preparación de las muestras, para luego realizar las pruebas correspondientes. Finalmente se analizaron y compararon los resultados obtenidos.

\section{RESULTADOSY DISCUSIÓN}

La caracterización de las muestras procedentes de dos sitios diferentes, perteneciente a la ciudad de Bogotá D. C., fue realizada en el Laboratorio de
Suelos del Instituto Geográfico Agustín Codazzi -IGAC-, en sus respectivas metodologías [23].

Las figuras 6 a 8 muestran el aspecto de los materiales recogidos, con la identificación establecida por el IGAC, resultados que se aproximan a lo expuesto según la clasificación RILEM [16] de los agregados reciclados en tipo I, tipo II y tipo III.

\subsection{Análisis químicos}

Una vez analizada la caracterización de los agregados reciclados de las matrices, cerámica y concreto, a partir de varias experiencias, se puede sugerir el uso de los escombros procesados de la ciudad en la fabricación de bloques de concreto, simples sin función estructural, pues los reportes de las muestras guardan una estrecha proximidad con los 


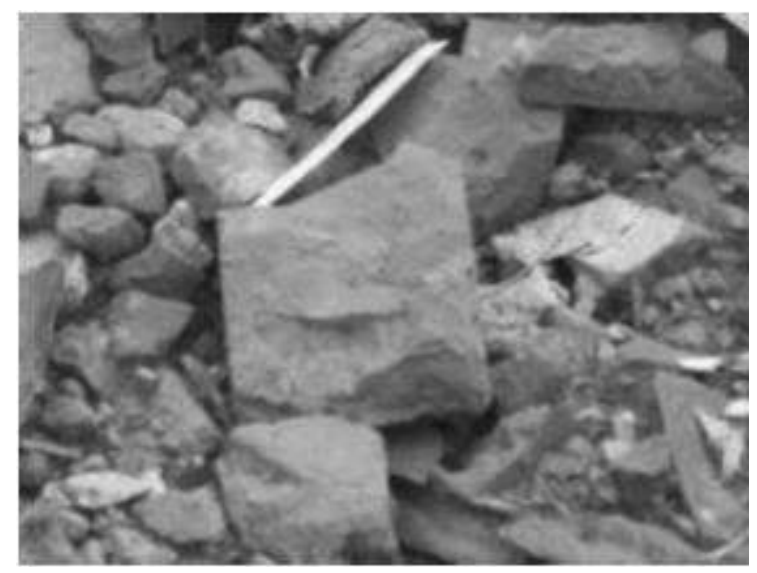

Figura 6. Tipo I/ No. 3-88879. Agregados procedentes de escombros de albañilería y/o mampostería Fuente: Elaboración propia

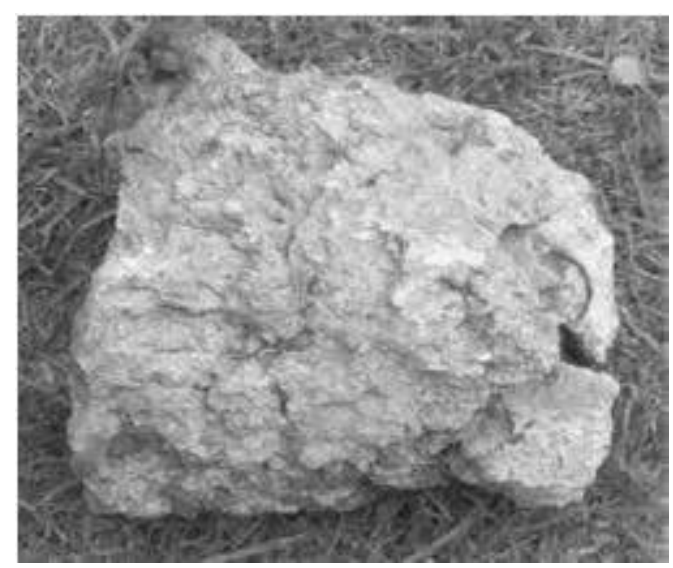

Figura 7. Tipo II / No. -88878. Agregados procedentes de escombros de concreto Fuente: Elaboración propia

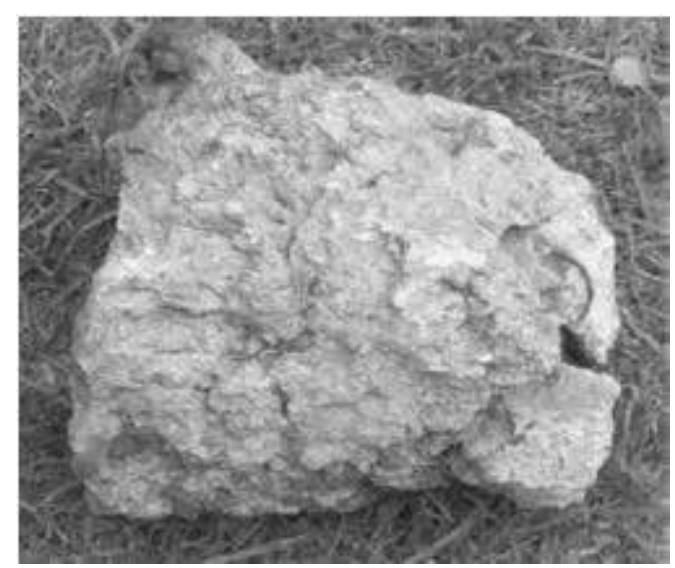

Figura 7. Tipo II / No. -88878. Agregados procedentes de escombros de concreto Fuente: Elaboración propia resultados suministrados en el estudio practicado en Campinas, Brasil [5].

Para diferentes autores, la determinación del pH es muy importante en las muestras de agregados reciclados, ya que nos indica el rendimiento y la adhesión de estabilización entre partículas, la cual se puede obtener en una solución de electrolitos con $50 \mathrm{~g}$ de material (arcilla, agregados o una mezcla de los mismos), expedido por el tamiz ABNT\#10, sometida a la agitación en una rotación de 250 rpm, en un tiempo de mezclado de 30 minutos, considerando una temperatura de $25^{\circ} \mathrm{C}$ [5]. En los estudios revisados y la muestra local, la basicidad de los agregados a base de concreto supera la de los cerámicos. Claro está, que valores de $\mathrm{pH}$ superiores a 11 dan por sentado un alto índice de calizas, baja actividad biológica, escasa concentración de nitrógeno, tanto como aportes de cal y cenizas.

Para todos los ensayos, el CO da indicio de la presencia de materiales orgánicos en las muestras, revalidando la condición inerte de los agregados reciclados. En la mayoría de los casos estudiados el porcentaje de $\mathrm{CO}$ es bajo, como se evidencia en la Figura 9.

Por su parte, los ensayos de los metales Al, $\mathrm{Pb}, \mathrm{Cd}$, Ni y $\mathrm{S}$, varían significativamente, como se muestra en la Figura 10. Los valores elevados de $\mathrm{Pb}$ suponen los aportes indiscriminados de materiales altamente tóxicos al medioambiente, no sólo en forma sólida, sino en forma de lixiviados, los cuales pueden alcanzar fuentes hídricas y acuíferos.

Aunque la desviación estándar no muestra diferencias significativas en todos los ensayos practicados, los niveles de $\mathrm{Mn}$ y Fe (mg/ $\mathrm{kg}$ ) en las dos matrices difieren notablemente y el nivel de $\mathrm{P}$ de los agregados cerámicos supera en casi $98 \%$ la muestra de concreto.

\subsection{Análisis físicos}

El análisis físico de los agregados locales analizados permitió concluir que son útiles para la fabricación de elementos estructurales sin función estructural, 
Tabla 4. Compilación de métodos fisicoquímicos para agregados

\begin{tabular}{|l|l|c|c|}
\hline \multicolumn{1}{|c|}{ Producto o material } & \multicolumn{1}{|c|}{ Método de ensayo } & Unidad & Norma \\
\hline Agregados & Absorción de Agregados Finos & $\%$ & INV E-222-07 \\
\hline Agregados & Absorción de Agregados Gruesos & $\%$ & INV E-223-07 \\
\hline $\begin{array}{l}\text { Prefabricados de } \\
\text { mampostería }\end{array}$ & Absorción & $\%$ & NTC 4017-05 \\
\hline Agregados & Peso volumétrico o Densidad Aparente & $\mathrm{kg} / \mathrm{m}^{3}$ & NTC 4017-05 \\
\hline Agregados & Resistencia al desgaste de agregados gruesos mayores a 19 mm & $\mathrm{mm}$ & NTC 93-1995 \\
\hline Agregados Finos & Equivalente de Arena & $\%$ & INV E-133-07 \\
\hline Agregados & Resistencia al ataque de Sulfatos -SO3- & $\%$ & NTC 126 \\
\hline Agregados & Silicatos (materiales cerámicos) & $\%$ & - \\
\hline $\begin{array}{l}\text { Prefabricados de } \\
\text { mampostería }\end{array}$ & Resistencia a la compresión (7 y 28 días). & $\mathrm{kg} / \mathrm{cm}^{2}$ & $\begin{array}{c}\text { NTC 4017-05 } \\
\text { NSR-98 }\end{array}$ \\
\hline Agregados & Granulometría (\% Arena, \% Grava) & $\%$ & NTC-77 1994 \\
\hline Agregados & Contenido de materia orgánica. Método ignición / color & - & $\begin{array}{c}\text { INV E-121-07 } \\
\text { NTC 127 }\end{array}$ \\
\hline Agregados & Elementos Menores (Mn, Fe, Zn, Cu y B, Pb*, Cd, Ni, Al). & $\%$ & - \\
\hline
\end{tabular}

Fuente: elaboración propia

al igual que en Brasil, ya que los porcentajes de absorción son favorables y análogos a los que presentan los agregados tradicionales (entre el $5 \%$ y el $15 \%$ ). Se esperaba contar con valores inferiores al $5 \%$ en los agregados de concreto, y se obtuvo un valor de 4,5\%. Por normativa INV E-222-07 e INV E-223-07, el porcentaje de absorción debe oscilar entre $7,0 \%$ y $13,5 \%$, mientras la resistencia a la comprensión debe superar los $35 \mathrm{~kg} / \mathrm{cm}^{2}$. La absorción y resistencia a la compresión para un tiempo de fraguado de 28 días, expuestas en la Figura 11, pueden inquirir el uso en elementos de mobiliario urbano, andenes y bloques, sin función estructural.

\section{CONCLUSIONES $Y$ RECOMENDACIONES}

Los agregados reciclados, para ser usados en diseños de mezcla de concretos (colocación de bloques, ladrillos, terminaciones u otras), deben cumplir con los requisitos de la normativa vigente, en el ámbito nacional las normas ICONTEC e INVIAS, que permiten distinguir los agregados reciclables y los no reciclables, tal como lo sugiere la metodología aplicada que incluye la implementación de operaciones unitarias y el uso posterior de ensayos de laboratorio.

Países como Irlanda, Nueva Zelanda, España, Estados Unidos, Brasil y México, actualmente cuentan con modelos y planes de manejo integral para este tipo de residuos, experiencias que estimulan el compromiso y el interés interadministrativo de los actores involucrados para la instalación de uni-
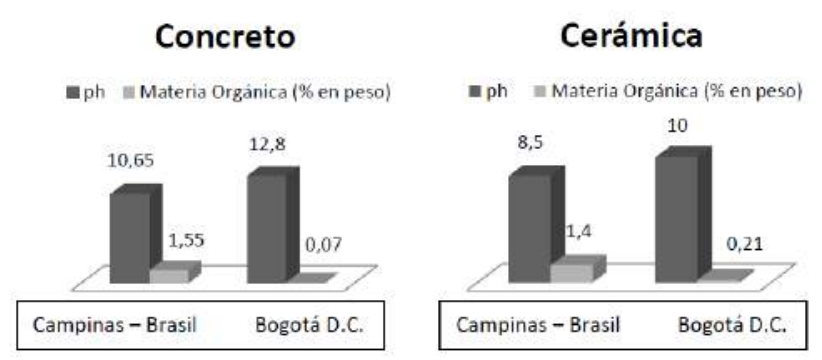

Figura 9. pH y \% Materia orgánica en agregados cerámicos y de concreto

Fuente: elaboración propia 
dades de aprovechamientos de escombros, fijas y/o móviles, capaces de producir piezas de mobiliario urbano, andenes, y viviendas prefabricadas.

Según estudios previos realizados en Colombia, en promedio el $55 \%$ de los RCD contiene agregados potencialmente reciclables, aproximadamente $60 \%$ de materiales a base de concreto, cerámicos y ladrillo (tipo I, tipo II, respectivamente, de acuerdo con la clasificación RILEM), al cual le siguen en menor proporción los materiales orgánicos y plásticos. Son, además, potencialmente apropiados, según la normativa internacional vigente, para convertirse en una fuente alternativa de materia prima en la fabricación de infraestructura urbana, y lograr beneficios económicos y ambientales, sin mermar la calidad técnica del producto terminado.

Si se comparan las especificaciones nacionales previstas por el ICONTEC, con los resultados de Campinas-Brasil, resulta viable la fabricación de bloques a base de materiales reciclables. Los porcentajes de absorción y la resistencia a la compresión, para un tiempo de fraguado de 28 días,

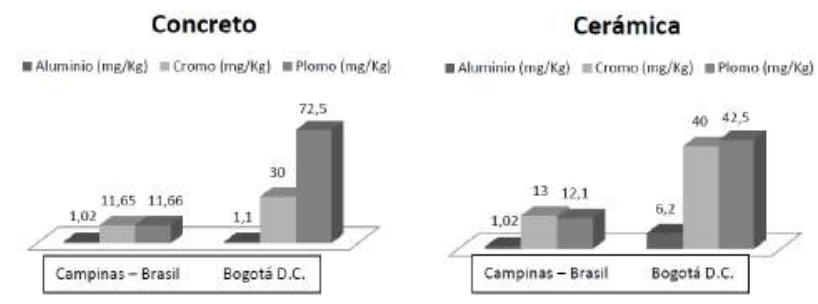

Figura 10. $\mathrm{Al}, \mathrm{Cr}$ y $\mathrm{Pb}$ en agregados cerámicas y de concreto

Fuente: elaboración propia

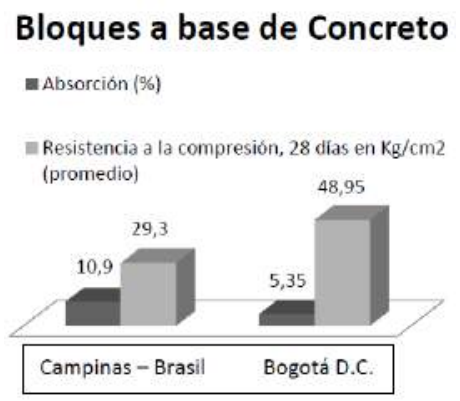

Figura 11. Comparación de resultados para bloques a base de concreto Fuente: elaboración propia se encuentran dentro de los límites permisibles de las Normas Técnicas Colombianas: NTC 4017, Métodos para muestreo y ensayos de unidades de mampostería y otros productos de arcilla; NTC 247, Bloques huecos de concreto para muros; NTC 673, Ensayo de resistencia a la compresión de cilindros normales de concreto y NTC 3546, Métodos de ensayo para determinar la evaluación en laboratorio y en obra, de morteros para unidades de mampostería simple y reforzada. Aunque según las normas INVIAS el porcentaje de absorción no alcanza el rango establecido, al estar en 4,5\%, fue cercano a lo esperado.

\section{REFERENCIAS}

[1]. ICPC. Instituto Colombiano de Producción de Cemento. [En Línea], Acceso 2011, Disponible: www.icpc.org. $\mathrm{co} /, 2005$.

[2]. LA REPÚBLICA. "Latin American Markets". [En Línea], Acceso Julio de 2010, Disponible: http://www.latinamerican-markets.com/colombia---consumo-de-cemento

-de Latin American Markets, 2005.

[3]. Gobierno Nacional De Colombia. Ley orgánica del plan de desarrollo, Ley 152 de 1994 (Julio 15). Diario Oficial N. 41.450 del 19 de julio de 1994, 1994.

[4]. Gobierno Nacional De Colombia. Ley de servicios públicos domiciliarios, Ley 142 de 1994. Diario Oficial 41.433 del 11 de julio de 1994, 1994.

[5]. Á. Chávez, Uso de lodo de estación para tratamiento de agua y agregado reciclado para la fabricación de elementos de albañileria. Campinas, Brasil: Universidad Estatal de Campinas. Ingeniería Civil, 2007.

[6]. 3. Velasco, L. Formulación de una propuesta de gestión ambiental para la recuperación y reciclaje de materiales de construcción y demolición. Tesis Administrador del Medio Ambiente. Universidad Tecnológica de Pereira. Facultad de Ciencias Ambientales. Programa Administración del Medio Ambiente. 2010.

[7]. Grupo de Investigación Estructuras y Edificación. España: Colegio de Ingenieros de Caminos, Canales y Puertos, 2007.

[8]. UAESP. "Caracterización de escombros por peso y por volumen en la ciudad”. Bogotá, D.C.: Unidad Administrativa Especial de Servicios Públicos, 2009. 
[9]. F. Prieto y J. Alonso. "Reciclaje de escombros de la construcción: una alternativa ecológica para México". Argentina: Revista de Ingeniería Sanitaria y Ambiental, vol. 93, pp. 94-101, 2007.

[10]. M. Toscano. Gestión de la ciencia y tecnología para el reciclado de los desechos sólidos en la construcción. Villa Clara, 2008.

[11]. J. Parra et al. Agregados reciclados para hormigón. Prueba Industrial. Disponible: http://www.minas.upm.es/ jornadas/III-JORNADAS-SAN\%20JUAN-ARGENTINA2003/COMUNICACIONES/Parra\%20y\%20 Alfaro,\%20Jose\%20Luis.pdf, 2003.

[12]. J. Rosa y E. Pastó. "Gestión de escombros y otros residuos de la construcción”. Revista Ambiente, pp. 53-56, 2004.

[13]. REBRI. Resource Efficiency in the Building and Related Industries. [En Línea], Acceso julio 2012, Disponible: http://www.rebri.org.nz/links/guidelines.html.

[14]. NCDWC. The National Construction and Demolition Waste Council, [En Línea], Acceso julio 2012, Disponible: http://www.ncdwc.ie/, 2003.

[15]. Agencia de Residuos de Cataluña, Generalitat de Catalunya, [En Línea], acceso 2012; Disponible: http:// www20.gencat.cat, Septiembre 2009.

[16]. RILEM. International Union of Laboratories and Experts in Construction Materials, Systems and
Structures, [En Línea], Disponible: http://www.rilem. net, 2006.

[17]. M. Serrano y D. Pérez. "Propuesta De Un Programa De Gestión Integral De Escombros". II Simposio Iberoamericano de Ingeniería de Residuos, Barranquilla, 2009.

[18] Velasco, L. Formulación de una propuesta de gestión ambiental para la recuperación y reciclaje de materiales de construcción y demolición. Tesis Administrador del Medio Ambiente. Universidad Tecnológica de Pereira. Facultad de Ciencias Ambientales. Programa Administración del Medio Ambiente. 2010.

[19] Guarín, N.; Montenegro, L.; Walteros, L.; Reyes, T. Estudio Comparativo en la Gestión de Residuos de Construcción y Demolición en Brasil y Colombia. Revista Gestión Integral en Ingeniería Neogranadina. Colombia. 2011.

[20]. J. Ramírez. "Guía para el manejo de residuos sólidos generados en la industria de la construcción”. México: Universidad de las Américas Puebla, 2007.

[21]. ICONTEC. Instituto Colombiano de Normas Técnicas y Certificación. [En Línea], acceso 2012; Disponible: http://www.icontec.org.co, 2007.

[22]. INVIAS. Instituto Nacional de Vías. [En Línea], acceso 2013; Disponible: http://www.invias.gov.co/, 2013.

[23]. IGAC. Instituto Geográfico Agustín Codazzi. Resultados Análisis de Laboratorio. Bogotá, D. C. 2012. 
